\title{
On-orbit passive thermography
}

\author{
PATRICIA A. HOWELL*, WILLIAM P. WINFREE, AND K. ELLIOTT CRAMER \\ Nondestructive Evaluation Sciences Branch \\ MS 231 NASA Langley Research Center \\ Hampton, VA 23681, USA
}

On July 12, 2006, British-born astronaut Piers Sellers became the first person to conduct thermal nondestructive evaluation experiments in space, demonstrating the feasibility of a new tool for detecting damage to the reinforced carbon-carbon (RCC) structures of the Shuttle. This new tool was an EVA (Extravehicular Activity, or spacewalk) compatible infrared camera developed by NASA engineers. Data was collected both on the wing leading edge of the Orbiter and on pre-damaged samples mounted in the Shuttle's cargo bay. A total of 10 infrared movies were collected during the EVA totaling over 250 megabytes of data. Images were downloaded from the orbiting Shuttle to Johnson Space Center for analysis and processing.

Results are shown to be comparable to ground-based thermal inspections performed in the laboratory with the same type of camera and simulated solar heating. The EVA camera system detected flat-bottom holes as small as $2.54 \mathrm{~cm}$ in diameter with $50 \%$ material loss from the back (hidden) surface in RCC during this first test of the EVA IR Camera. Data for the time history of the specimen temperature and the capability of the inspection system for imaging impact damage are presented.

Keywords: Thermography; Shuttle; impact damage; RCC; composites

\section{Introduction}

After the Shuttle Columbia accident, several new tools and inspection procedures were developed to ensure the integrity of the Shuttle's thermal protection system prior to reentry. The development of a nondestructive, noncontacting, handheld EVA inspection tool was proposed as an additional focused inspection technique. The goal of this tool is to provide information regarding the presence of subsurface damage to the reinforced carboncarbon (RCC) material of the wing leading edge of the Shuttle. Thermography had been used extensively during ground tests to quantify impact-induced damage to the orbiter's wing leading edge material as part of NASA's Return to Flight program. 
Based on the success of thermal NDE for ground inspections of Shuttle RCC, a handheld infrared camera was developed to test the ability of thermography to detect possible damage during a mission. To save on weight and power requirements, a commercially available microbolometer was modified to withstand the space environment, with special consideration given to the constraints of using the camera during an EVA. This instrument is a compact, low-mass, low-power solution for TPS inspection.

To detect underlying RCC damage with infrared thermography, a thermal gradient through the RCC panel must exist. On the ground, inspectors use large flash lamps to generate a thermal gradient. The power, weight and cost requirements for space-qualifying flash lamps for the on-orbit system made their development impractical. Rather than packaging a flash lamp for space, the EVA IR Camera project team conducted a series of tests on the ground to demonstrate that sunlight and shadowing could be used to generate an adequate thermal gradient through the RCC panels. This significantly simplifies the development tasks and reduces project costs. However, it also increases operational complexity, since crewmembers are constrained to inspecting the RCC in sunlight.

The flight experiments were designed to determine how well the technique would work onorbit, where the constraints of space flight and the thermal environment challenged the technique and required the development of space-hardened flight hardware. If successful, the EVA IR Camera would provide information regarding the extent of subsurface damage to the RCC.

Given the urgency with which the program needed to implement the use of the EVA IR Camera for RCC inspection, together with the immaturity of using infrared thermography in space for RCC damage detection, the Space Shuttle Program (SSP) directed the project to limit its initial scope to building flight units certified as safe to test the application of infrared thermography for on-orbit RCC inspection rather than to building flight units certified to detect specific RCC damage.

This report delineates the major differences in boundary conditions that effect the measurements between the thermography inspections conducted on the ground versus the conditions and limitations inherent on-orbit, and how those differences were managed to provide a potential inspection capability during a mission. Processing algorithms were developed to correct for the motion between the object and the camera. Since a microbolometer has significant associated noise, an algorithm was added to the processing that spatially smoothes the data while maintaining information along defect edges. This algorithm is shown to substantially improve the image quality for this detector. The boundary conditions existing during orbit for both heating and cooling are significantly different than those existing for ground inspections. These differences and their effect on the inspection technique are discussed. 


\section{Hardware and Measurement Technique Development}

\subsection{Camera Hardware}

The thermal NDE system used for ground inspections consists of a high resolution, high speed cooled detector. The significant limitations of available resources during space missions, such as weight and power, were defining parameters in the selection of the infrared camera. Further, budget and time considerations led to a decision to modify a COTS camera. This camera had to be capable of collecting and storing a series of images to provide the necessary data for NDE processing. Details of the camera modifications necessary to transition the COTS camera to space flight hardware are discussed in "Development of an extra-vehicular (EVA) infrared (IR) camera inspection system."

An uncooled microbolometer camera was chosen in order to minimize the system weight and required power. While cooled detector cameras provide significantly better signal-tonoise, the microbolometer provides sufficient signal-to-noise for detecting delaminations of the size and depth of interest, with lower weight and power consumption. The camera has a spectral range of 7.5 to $13 \mu \mathrm{m}$ with a $320 \times 240$ pixel focal plane array. The field of view is $24^{\circ} \times 18^{\circ}$ with a minimum focus distance of $0.3 \mathrm{~m}$. It has a thermal sensitivity of $0.06^{\circ} \mathrm{C}$ at ambient and can collect and store up to 600 frames of 14 bit integer data to builtin RAM at frame rates varying from 0.6 hertz to 30 hertz. At 0.6 hertz, a maximum of 16.67 minutes of data can be recorded to internal camera memory. At 30 hertz, 20 seconds of data can be recorded before the memory buffer is filled and the data must be transferred to the camera flash memory card. A 1 GB memory card is stored in the camera to allow multiple infrared movies to be permanently saved. The memory card can store up to 10 full memory buffers, or 6000 frames of infrared data. The flash memory card is then used to transfer the data to an onboard computer for transmission to the ground for processing.

\subsection{Feasibility Testing}

To save development time and costs as well as system weight and required energy, the feasibility of detecting sub-surface damage under on-orbit solar conditions was explored by imaging damaged RCC samples using quartz lamps to simulate the expected solar flux. The maximum expected absorbed solar flux on-orbit is approximately $1100 \mathrm{~W} / \mathrm{m} 2$. Two quartz lamps were used in a laboratory environment (ambient temperature and pressure) to simulate expected solar flux. By measuring the temperature profile in RCC and knowing the material properties of RCC (thermal conductivity), the lamp power settings were calibrated to solar flux levels. In addition, the measured temperature profiles were compared to a model simulation of RCC and found to be in good agreement. Figure 1 shows the laboratory test setup. 


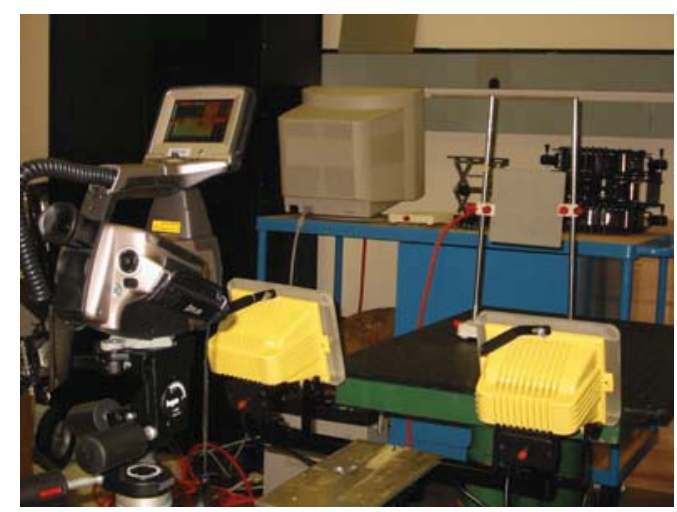

Figure 1. Laboratory test setup simulating solar flux conditions on RCC. Quartz lamps were calibrated to the expected solar flux.

\subsection{Thermal Boundary Conditions and Operational Considerations On-orbit}

Significant changes to the data acquisition and processing approach were necessary to transition the ground system to an on-orbit inspection system. The thermal boundary conditions on the RCC are significantly different, with no convective heat losses from the structure, but significant radiative heat transfer possible. Additionally, significant heat conduction across a delamination is possible for ground inspections however in the vacuum of space there is no air to conduct heat across the gap.

Operationally, issues such as focus, frame rate acquisition time, issues of uneven heating and correction for the motion between the astronaut and the test article were investigated. To test these changes in the operational system, a set of seven RCC panels were obtained and impacted with varying velocities of SOFI (Spray-On Foam Insulation) foam to create samples with varying degrees of damage.

\subsection{Impact Panel Descriptions}

Seven samples approximately $15 \mathrm{~cm}$ wide x $15 \mathrm{~cm}$ high of RCC material were impacted with SOFI foam as shown in Figure 2. All seven samples were cut from a previously-flown section of RCC to provide samples with surface emissivity variations as seen on flight panels. The samples were held in a metal frame around the edge to allow the RCC to flex upon impact. The edges of the samples were cushioned from the metal frame with pliable putty to allow further sample motion. The RCC was impacted with SOFI foam that measured $2.5 \mathrm{~cm} \times 2.5 \mathrm{~cm}$ cross section and was $5 \mathrm{~cm}$ long with a weight of 2 grams. The panels were impacted at speeds of approximately $2000 \mathrm{ft} / \mathrm{s}$. Multiple impact events were conducted to create samples with more significant damage. 

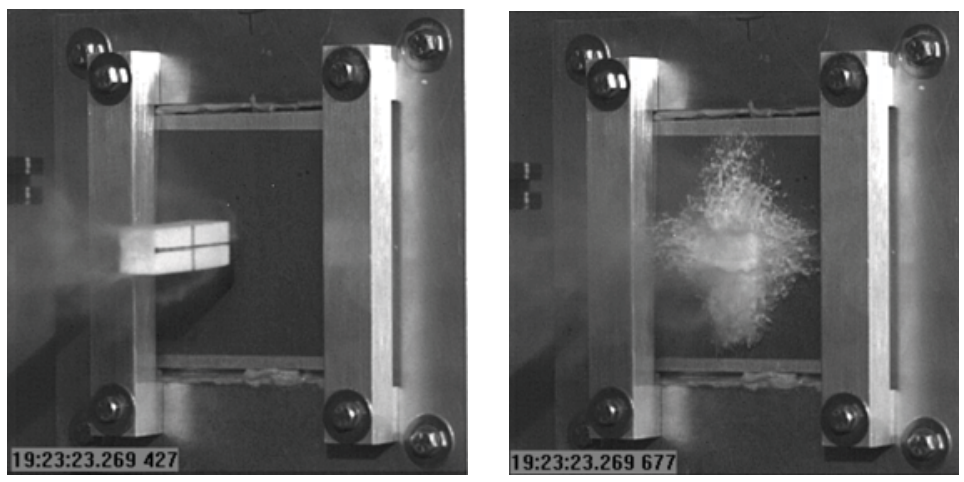

Figure 2. Impact of RCC with foam at approximately $2000 \mathrm{ft} / \mathrm{s}$. Left-hand image shows block of foam just prior to impact. Right-hand side shows foam immediately after impact. The RCC panels are 6 in. x 6 in. and were mounted to allow in-plane deflection of the RCC upon impact. The foam impact coupons are $2.5 \mathrm{~cm} . \mathrm{x}$ $2.5 \mathrm{~cm} . \mathrm{x} 7.6 \mathrm{~cm}$.

These seven impact panels (see Figure 3) provided samples with a range of realistic delaminations to be used for technique development. The samples were characterized with ground-based flash thermography, with both active and simulated solar heating, and with both the ground-based IR camera and a commercial version of the microbolometer camera chosen for the orbital IR camera.

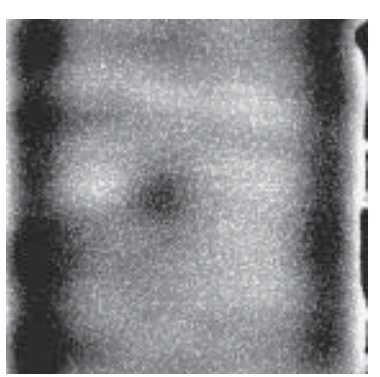

Impact sample \#1

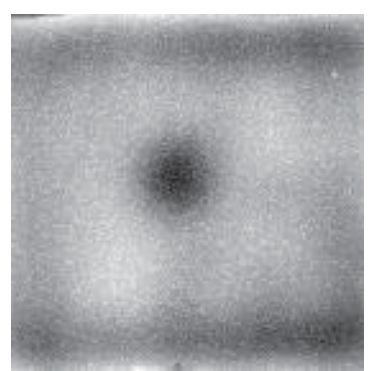

Impact sample \#2

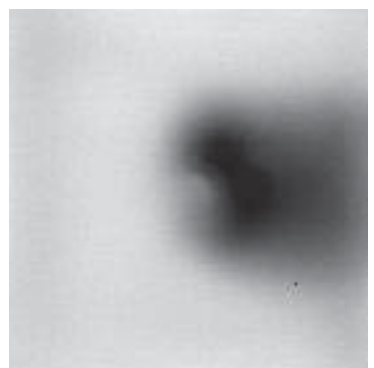

Impact sample \#3

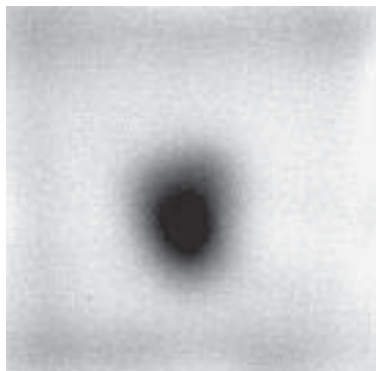

Impact sample \#4

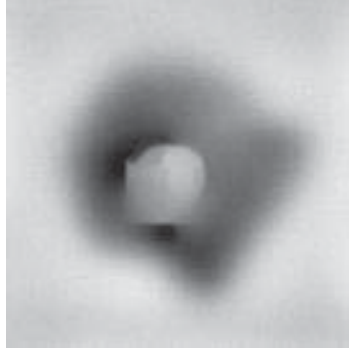

Impact sample \#6

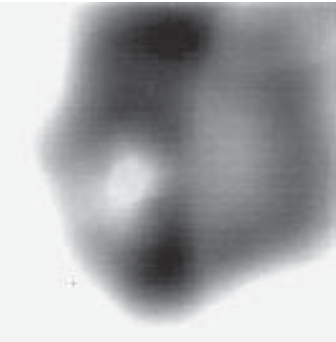

Impact sample \#7

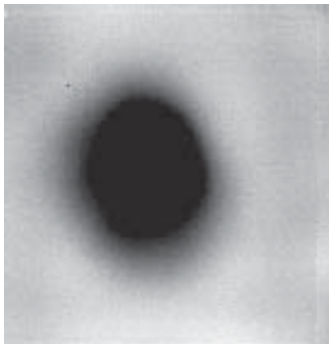

Impact sample \#8

Figure 3. Processed flash thermography (baseline) data of seven impacted RCC samples. Delaminations from $1.27 \mathrm{~cm}$ diameter (\#1) to $11.5 \mathrm{~cm} \mathrm{x} 14 \mathrm{~cm}$ (\#7) (Note: impact sample \#5 was not part of the test set.) 


\subsection{Input Heat Flux.}

One of the requirements of transitioning the NDE technique to an orbital system is the adaptation of the input heat flux for in-space operations. For the thermal system used for ground RCC tests, a short flash of heat is delivered to the front surface of the sample via two $4800 \mathrm{~J}$ xenon flash lamps. The infrared data is acquired during the cool-down phase. Reliance on solar energy to provide the input heat flux results in a much slower application of heat and a slower cooling rate. Quantitative data reduction techniques that are used for RCC inspection, a Principal Component Analysis technique, require a time-varying thermal contrast between a flawed and an unflawed material. On-orbit, this condition is best achieved by data acquisition at the onset of solar heating or at the end of solar heating phase. Rather than relying on the orbital cycle to set the timing of the solar heating cycle, blocking the sunlight controls the initiation and end of solar heat during data collection. Ground-based experiments showed the feasibility of this approach both from a crew operations perspective as well as a data analysis perspective. For this test, two RCC specimens with known defects were placed under solar heating conditions. The first sample measured approximately $5 \mathrm{~cm} \mathrm{x} 15 \mathrm{~cm}$ and contained three full-width defects of various depths. The second sample was approximately $15 \mathrm{~cm}$ x $15 \mathrm{~cm}$ and contained numerous flat-bottom holes of various depths and diameters. A photograph of these samples is shown in Figure 4. NDE results of data collected under solar conditions on RCC both with and without shading are shown in Figure 5.

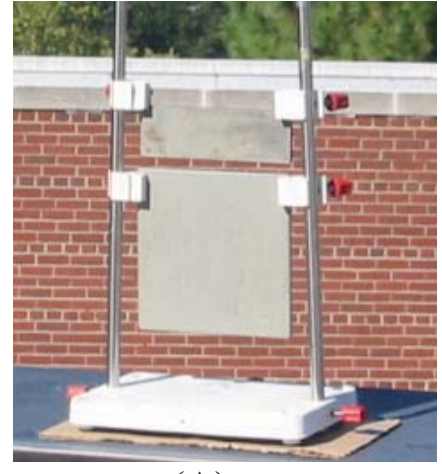

(A)

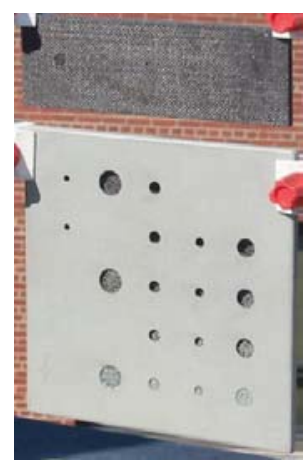

(B)

Figure 4. Photograph of (A) front of samples and (B) back of RCC samples used for testing of thermal technique using solar heating
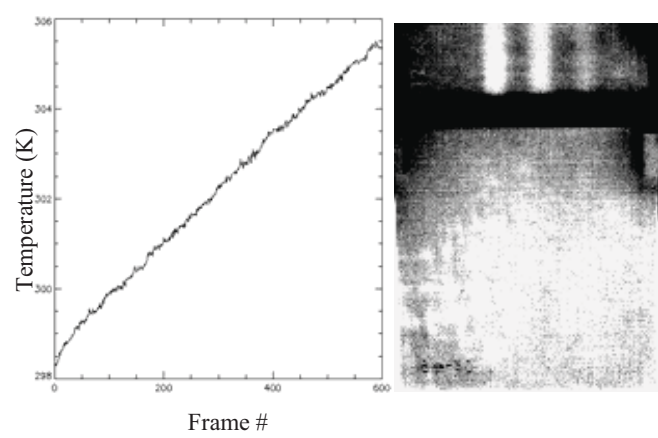
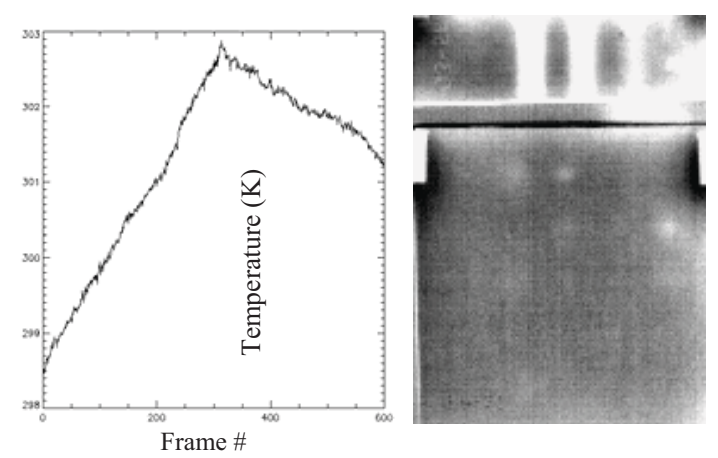
Figure 5. (A) Processed thermal data on Shuttle RCC under solar conditions with no shading and (B) with shading

\subsection{Data analysis}

Principle Component Analysis (PCA) is an algorithm based on decomposition of the thermal data into its principle components. Singular value decomposition is a routine used to find the singular values and corresponding eigenvectors of a matrix. Since thermal NDE signals are well-behaved and slowly decaying waveforms, the spatial variations of the entire data set is usually contained in the first and second eigenvectors and accounts for most of the data variance. PCA analyses were used routinely on the flash thermography conducted for ground inspections, and is discussed in greater detail elsewhere. It was found that PCA also works well for the longer heat pulse input flux condition, using approximately the first 10 seconds of data immediately after shadowing of the sample.

Anistropic Diffusion. Anisotropic diffusion is a data processing technique that has been shown to enhance the contrast between damaged and undamaged regions in thermal images. The technique has been shown to significantly reduce spatial image noise while maintaining defect contrast and preserving the important features of a flaw. This is accomplished by a convolution of the data with a function containing a diffusion parameter. This parameter is high in regions of low gradients in image intensity and low in regions of high discontinuities in image intensity, as is the case near defect edges. For this data, the algorithm was applied to each frame of the data collected to remove spatial noise from the microbolometer data while maintaining any signal due to a delamination. Application of this algorithm resulted in a significant improvement in signal-to-noise of the final post-processed data, as seen below in Figure 6.

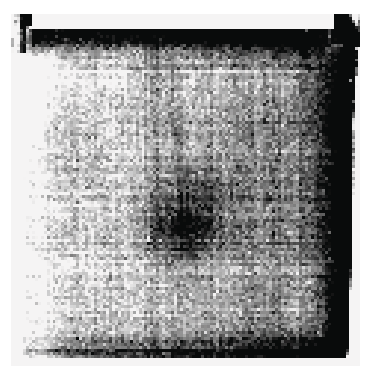

(A)

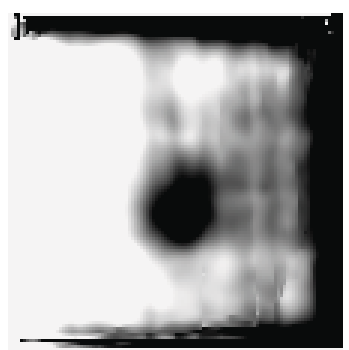

(B)

Figure 6. Post-processed microbolometer data, solar heating (A) without anisotropic diffusion, and (B) with Anisotropic Diffusion algorithm applied. 


\subsection{Frame rate.}

To minimize transfer time from camera memory to the internal compact flash card, minimizing the number of frames of data collected is critical. Reducing the number of frames stored can be accomplished by decreasing the frame rate or by decreasing the amount of time data is collected. Operationally, the crew must coordinate the start of data collection with the application of a shadowing source during the sequence. For the flight experiment, this parameter was set at 60 seconds to ensure ample time for collection of the 10 second window after the initiation of shading.

Data was collected on impact panel number 8 , and subsampled to obtain different effective frame rates. After applying the anisotropic diffusion algorithm and PCA during the first ten seconds after initiation of shading, a signal-to-noise analysis was performed to determine the loss in signal due to decreasing the frame rate. The signal-to-noise is defined as the difference between the base material response and the flaw signal divided by the standard deviation of the base material. A gaussian fit of the histogram was used to determine the normal and standard deviation in the response of the base material. The histogram and its fit are shown in

Figure $7 \mathrm{~b}$. The lower shoulder was selected as the value for the flaw.

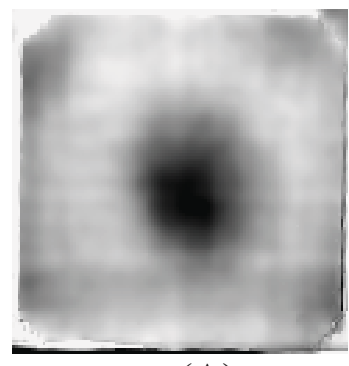

(A)

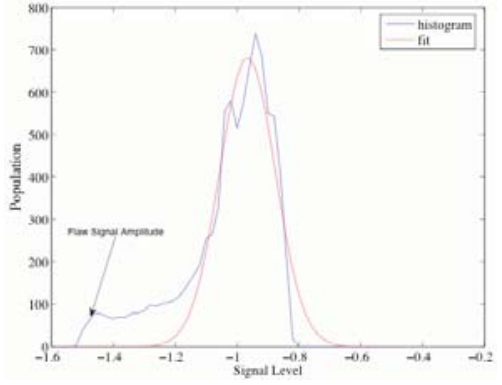

(B)

Figure 7. (A) Processed IR data, Impact Sample \#8 (B) Histogram of image A

As seen in the Table 1 below, a 30\% loss in signal-to-noise occurs when reducing the frame rate to $1 \mathrm{~Hz}$. For the DTO, data was collected at $5 \mathrm{~Hz}$ for 60 seconds, resulting in a transfer time to the compact flash card of approximately 2 minutes.

Table 1. Comparison of Signal-to-noise for various data collection rates

\begin{tabular}{|l|l|l|l|l|}
\hline Signal to Noise calculated for impacted specimen \\
\hline $\begin{array}{l}\text { Reduction parameters: } \\
\text { Frequency of } \\
\text { acquisition, start and } \\
\text { stop channels }\end{array}$ & Signal & $\begin{array}{l}\text { Standard Deviation } \\
\text { Base Response }\end{array}$ & $\begin{array}{l}\text { Signal to } \\
\text { Noise }\end{array}$ & $\begin{array}{l}\text { \%oss } \\
\text { from 15 } \\
\mathrm{hz}\end{array}$ \\
\hline $15 \mathrm{~Hz}-310,460$ & 0.50 & 0.09 & 5.5 & $\mathrm{X}$ \\
\hline $5 \mathrm{~Hz}-104,154$ & 0.50 & 0.09 & 5.5 & $0 \%$ \\
\hline $1 \mathrm{~Hz}-21,31$ & 0.39 & 0.10 & 3.9 & $29 \%$ \\
\hline
\end{tabular}




\begin{tabular}{|l|l|l|l|l|}
\hline $0.5 \mathrm{~Hz}-10,15$ & 0.34 & 0.10 & 3.4 & $38 \%$ \\
\hline $0.5 \mathrm{~Hz}-11,16$ & 0.37 & 0.10 & 3.6 & $34 \%$ \\
\hline
\end{tabular}

\subsection{Effects on measurement of environment - operating in a vacuum.}

A custom-made vacuum chamber was used (pictured in Figure 8a) to quantify the effect on the measurement and detectability of defects for data taken in the vacuum of space. The vacuum chamber has three windows. Two quartz windows provide access for sample heating from an external source. Alternatively, incident heat flux on the samples can be accomplished with IR heaters placed inside the chamber and affixed to the interior of the front wall. The center window is germanium for viewing with the infrared camera. The maximum vacuum achievable is approximately $10^{-8}$ torr. As can be seen by comparing Figure $8 \mathrm{~b}$ and Figure $8 \mathrm{c}$, a vacuum provides a noticeable increase in defect detectability. At atmospheric pressure, air in the delamination can result in significant heat transfer across a delamination, even when the delamination between the composite layers is well defined. In a vacuum the delamination more closely approximates a perfect insulator.

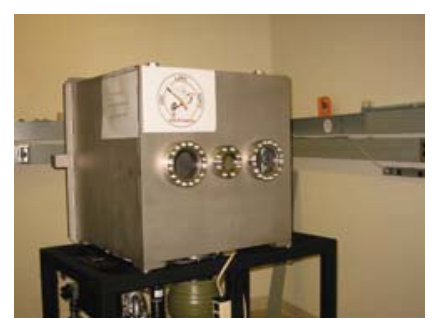

(A)

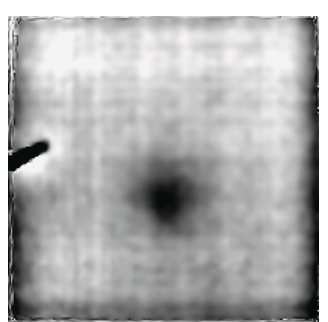

(B)

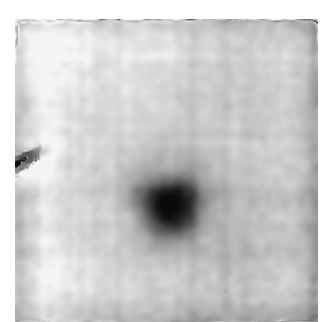

(C)

Figure 8. (A) Photograph of vacuum chamber $\quad$ (B) Panel \#4 at atm (760 torr) $\quad$ (C) Panel \#4 at vacuum (9x10-7 torr)

\subsection{Radiation losses}

A finite element simulation of the specimen with radiative heat transfer and a periodically applied heat flux to the front, and with continuous radiative heat transfer into fixed temperature at both the front and back surfaces was performed. A delamination was placed at the center plane of the specimen, with a radius-to-thickness ratio of 4:1. To reduce the computational time required for the simulation, the simulation was performed in cylindrical coordinates, approximating a circular delamination. For the simulations reported here, the back surface was assumed to always radiate into $270^{\circ} \mathrm{K}$ and the front surface to radiate into either $10 \mathrm{oK}$ or $170^{\circ} \mathrm{K}$. Simulations were also performed with incident fluxes of $1.1 \mathrm{Kwatt} / \mathrm{meter}^{2}$ and $0.55 \mathrm{Kwatt} / \mathrm{meter}^{2}$. For all cases considered here, the incident flux is applied for the first half of a 90 minute cycle. All simulations assume an initial temperature of the entire specimen is $290^{\circ} \mathrm{K}$, with two full cycles of simulation before beginning to record the results. This was found to give results that were independent of the initial condition. 
The time history of the temperature at a point far from the delamination is shown in Figure 9. The time in the figure is set to be zero at the beginning of a cycle. The simulation indicates the temperature increases at the beginning of the cycle as the incident flux is applied. During the application of the incident flux, the temperature increases at a decreasing rate, asymptotically approaching a temperature that is determined by the point when the incident flux would be equal to the radiative heat transfer. This is seen in the figure where the case of radiative heat transfer into $170^{\circ} \mathrm{K}$ approaches a slightly higher temperature than for radiative heat transfer into $10^{\circ} \mathrm{K}$.

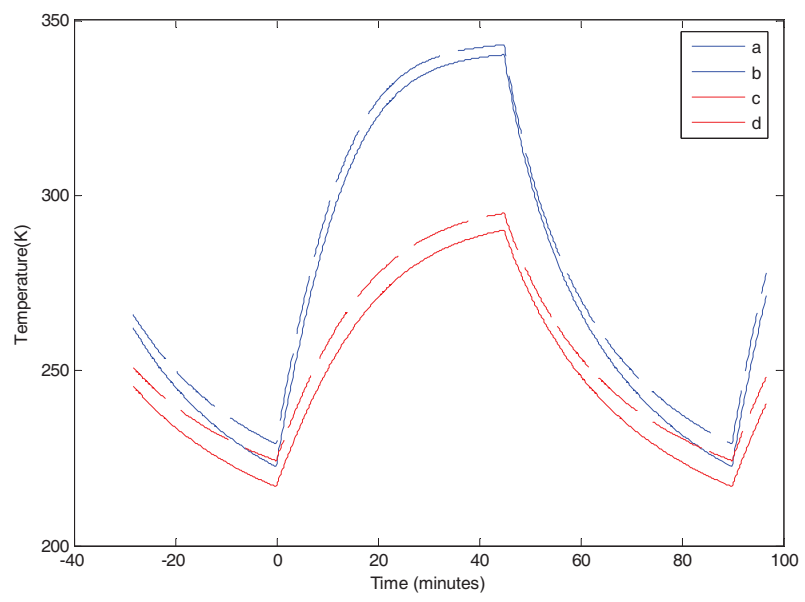

Figure 9. Results of simulation of thermal response of specimen radiating into two different temperatures and with two different surface fluxes. The individual plots are (a)- surface flux of $1.1 \mathrm{kw} / \mathrm{meter}^{2}$, radiating into $10^{\circ} \mathrm{K}$, (b) - surface flux of $1.1 \mathrm{kw} / \mathrm{meter}^{2}$, radiating into $170^{\circ} \mathrm{K}$, (c) - surface flux of $0.55 \mathrm{kw} / \mathrm{meter}^{2}$, radiating into $10^{\circ} \mathrm{K}$ and (d) - surface flux of $0.55 \mathrm{kw} /$ meter $^{2}$, radiating into $170^{\circ} \mathrm{K}$.

For detection of the delamination, the important characteristic is the temperature difference between the delamination and an undamaged region. The temperature difference between the center of delamination and a reference point 5 times the radius away from the edge of the delamination is shown in Figure 10. At the beginning of the cycle when the incident flux is applied, there is a rapid increase in the temperature over the entire delamination, relative to a non-delaminated point. This rapid increase lasts for a few seconds when the most significant thermal fluxes are surface normal. Following this stage, in-plane heat conduction limits the contrast between the center of the delamination and a nondelaminated reference point. The time and amplitude of the maximum contrast increase with the diameter of the delamination and decreases with the depth of the delamination. As the surface temperature increases, the radiative heat loss from the surface reduces total flux into the surface and the contrast decreases. However for the cases shown here, this is not a significant effect.

At the midpoint of the cycle, the incident flux becomes 0 , and the radiative heat transfer begins to cool the specimen. The temperature difference decreases rapidly, with the most significant decrease occurring within approximately 20 seconds. The absolute value of the temperature difference begins to decrease as the temperature of the specimen decreases. When the incident flux is zero, a state is reached where the temperature difference between 
the delaminated and undamaged regions depends on the difference in temperature between the temperature being radiated into by the front and back surfaces.

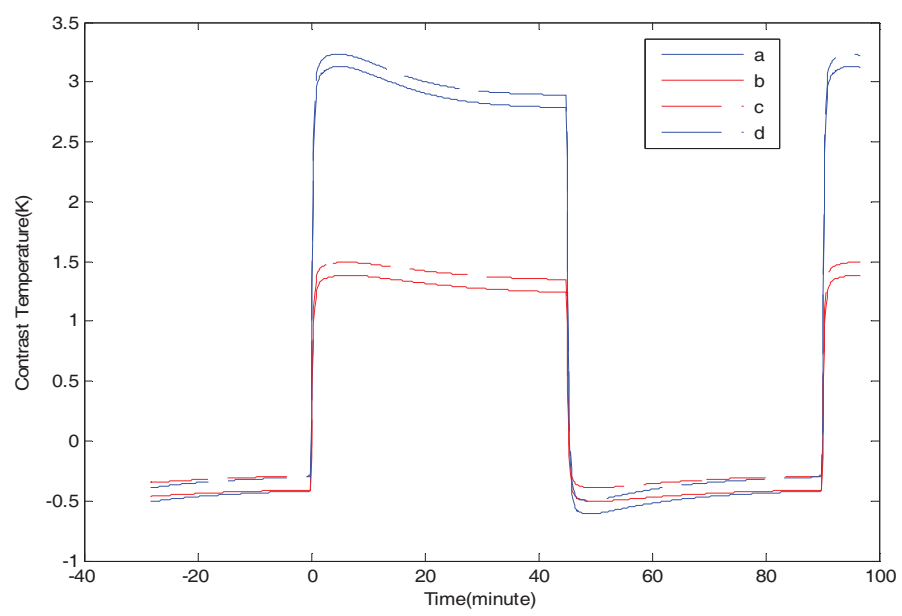

Figure 10. Contrast as a function to time based on simulation of thermal response of specimen radiating into two different temperatures and with two different surface fluxes. The individual plots are (a) - surface flux of $1.1 \mathrm{kw} / \mathrm{meter}^{2}$, radiating into $10^{\circ} \mathrm{K}$, (b) - surface flux of $0.55 \mathrm{kw} / \mathrm{meter}^{2}$, radiating into $170^{\circ} \mathrm{K}$, (c) - surface flux of $0.55 \mathrm{kw} / \mathrm{meter}^{2}$, radiating into $10^{\circ} \mathrm{K}$ and (d) - surface flux of $1.1 \mathrm{kw} / \mathrm{meter}^{2}$, radiating into $170^{\circ} \mathrm{K}$.

The time history of the spatial temperature profiles is shown in Figure 11 at the beginning of the cycle. Time in the figure is referenced to the beginning of the cycle, corresponding to the application of incident flux. As can be seen in the figure, the contrast rapidly develops. It should also be noted that the best definition as to the extent of the delamination is before there is any significant in-plane heat flow.

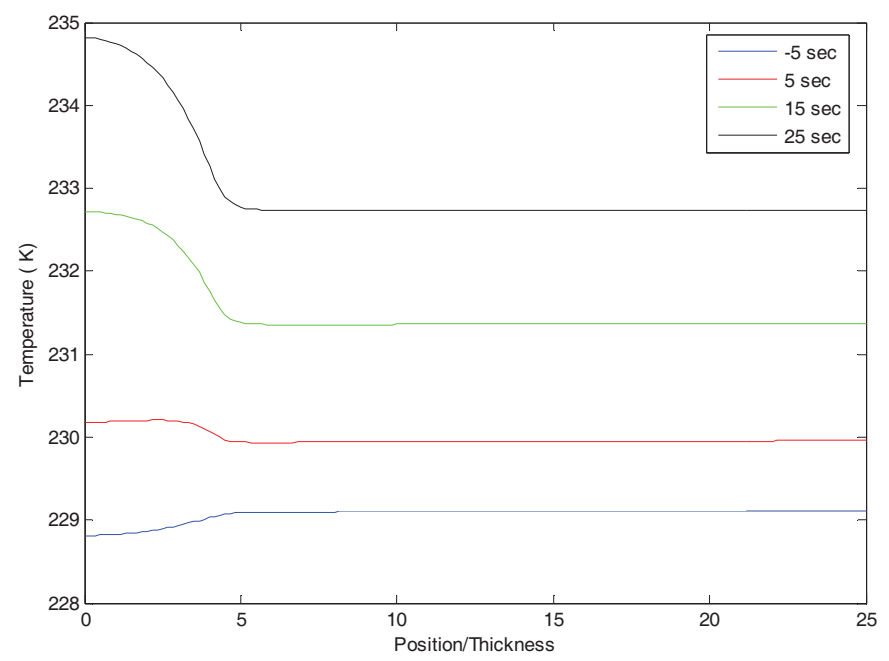

Figure 11. Temperature profile for different times based on simulation of thermal response of specimen radiating into $170^{\circ} \mathrm{K}$ different temperatures, with a surface flux of $1.1 \mathrm{kw} /$ meter $^{2}$. Time is relative to the start of application of the flux. 
The temperature profile for different times at the midpoint of the cycle is shown in Figure 12. This corresponds to the incident flux becoming zero with time given relative to the midpoint in the cycle. Again the profile changes rapidly with the temperature difference between the delamination and undamaged regions becoming negative within 45 seconds. The extent of the delamination is not as clearly defined in these profiles.

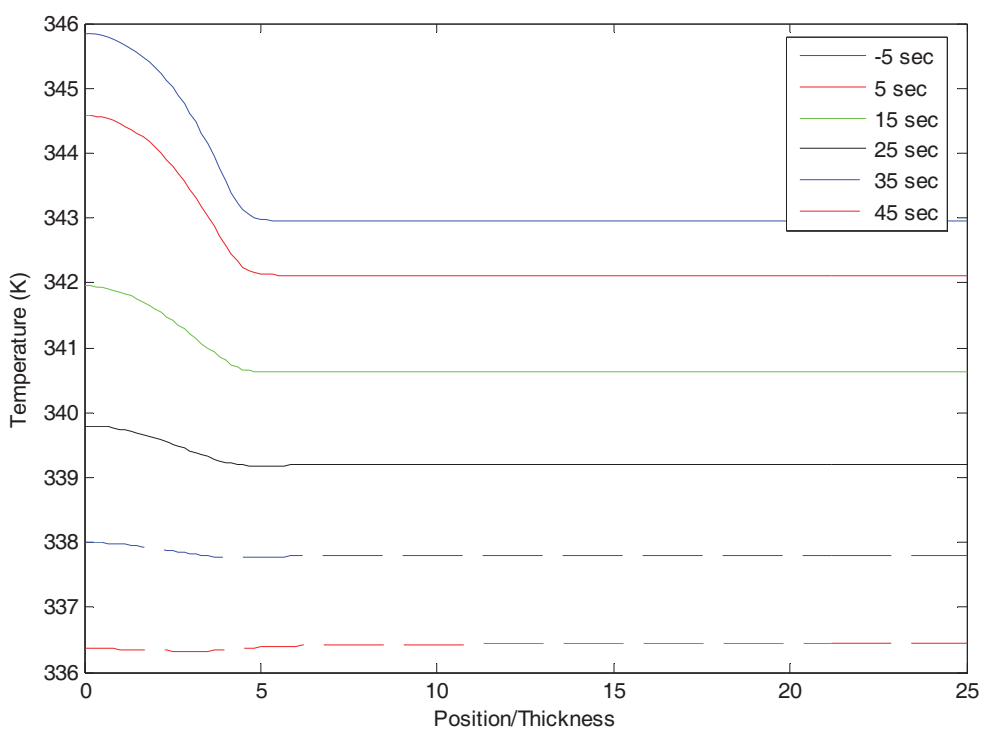

Figure 12. Temperature profiles for different times based on simulation of thermal response of specimen radiating into $170^{\circ} \mathrm{K}$ different temperatures, with a surface flux of $1.1 \mathrm{kw} / \mathrm{meter}^{2}$. Time is relative to the removal of the flux.

\section{Flight Results}

Two major flight test objectives were identified. The first objective was to verify the operational inspection technique, and obtain measurements of the temperature profile of RCC under on-orbit solar heating conditions. Data from these measurements would be compared to wing leading edge temperature predictions and to estimate the boundary conditions on the RCC important to a NDE inspection. These parameters are required to predict damage detection limits. The second objective was to illustrate the effectiveness of the NDE technique under orbital conditions, validating the ground predictions for the onorbit system. 


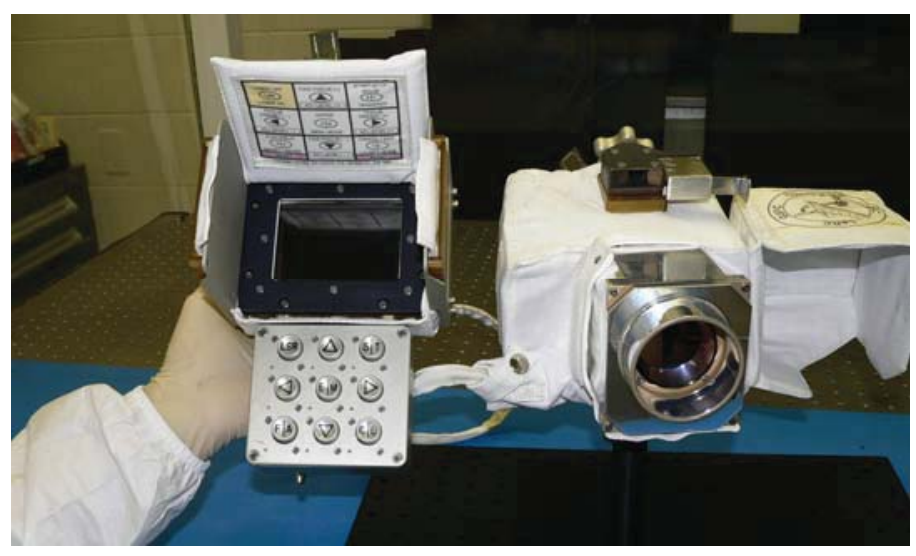

Figure 13. Photograph of EVA IR Camera Flight Unit. The camera consists of two major components: the camera body with the germanium infrared detector, battery, memory, and flash drive, and the Remote Control Unit (RCU) containing the LCD screen and control buttons.

\subsection{Flight Test Objective \#1:}

The first flight test objective was to obtain wing leading edge (WLE) temperature data during orbit for comparison to wing leading edge temperature predictions and estimate the thermal boundary conditions on the RCC important to the NDE inspection.

Two 20-second thermal movie segments of the starboard WLE were obtained on STS-121, as shown in Figure 14. One movie segment was taken just after egress from the airlock, and the other toward the end of the EVA. Constraints of EVA timeline planning were the primary driver to this schedule. Coincidentally, both of these thermal movie segments were taken at approximately the same relative point in the orbital day/night sequence approximately 5 minutes prior to orbital sunrise as shown below in Figure 15.
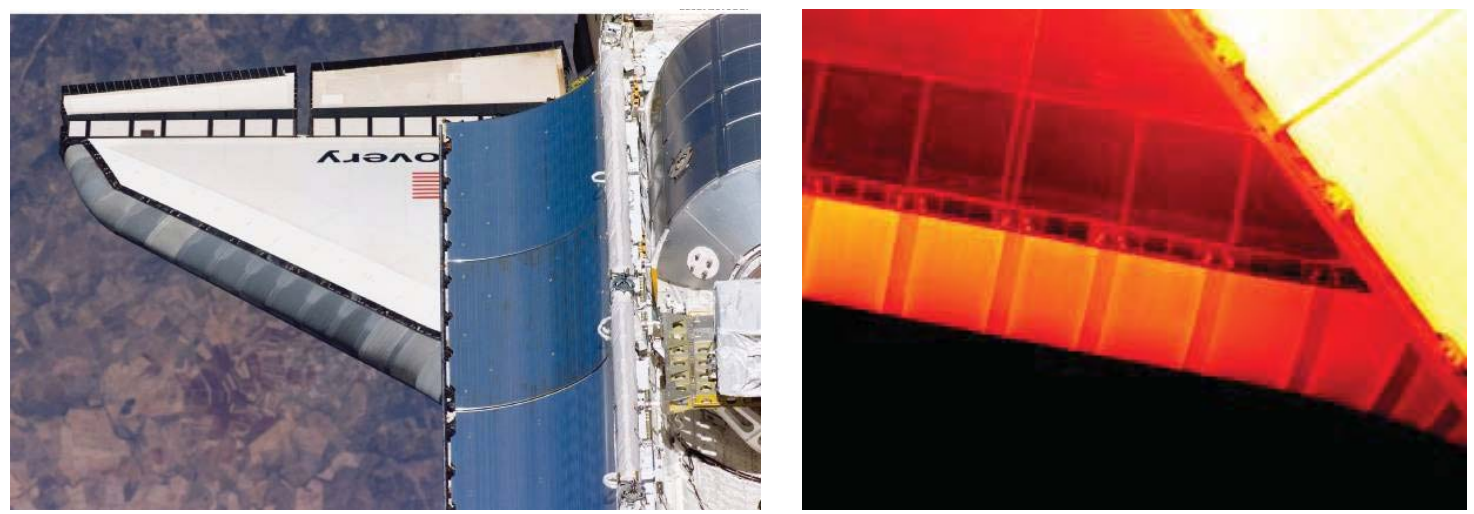

Figure 14. (A) Photograph of Shuttle Wing Leading Edge from approximate perspective of infrared imagery captured for Objective \#2. (B) Infrared Imagery from EVA IR Camera taken during STS-121. Temperature 
measurements were recorded at several times during flight to obtain boundary condition information for use in NDE simulations.

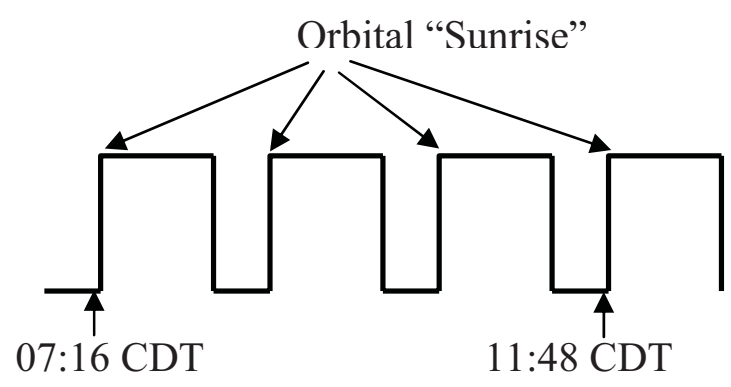

Figure 15. Graphic illustrating the periodic thermal solar load on the RCC during orbit overlaid with the times data was taken on the Shuttle wing leading edge with the EVA IR Camera on STS-121. Both datasets were recorded, coincidentally, just prior to sunrise.

Because the purpose of this data was to obtain WLE temperatures over a large span of the wing RCC panels, the astronaut was positioned approximately 20 feet from the wing. During an actual inspection, the proposed operational concept is to position the astronaut 3 to 6 feet away from the wing panel of interest. This distance is determined by the defect size of interest and the spatial resolution of the camera optics.

Figure 16 shows that for a region of interest over a representative RCC panel on the wing of the Shuttle and for a region over the white Nomex Felt Reusable Surface Insulation (FRSI) acreage region of the wing, the temperature has reached equilibrium in both regions during the nighttime cycle. The RCC is holding at a stable $-44^{\circ} \mathrm{C}$ and the FRSI is approximately $4^{\circ}$ colder at $-48^{\circ} \mathrm{C}$. Assuming the FRSI to be a thermal insulator, it is possible to approximate the background temperature for the radiative heat loss to space as $48^{\circ} \mathrm{C}$. The first infrared movie taken at 07:16 CDT July 15, 2006 was used in Figure 16. The second movie of the WLE taken 4.5 hours later of the same section of the wing had similar results, although the temperature of the FRSI was as much as $5^{\circ} \mathrm{C}$ colder than the previous movie sequence recorded and was observed to be slightly decreasing during the 20 second recording period. The difference in temperature is attributed to the change in the temperature of surround structures between measurements. Since this temperature difference does not significantly effect the radiation heat loss at the surface, it is does not appreciably impact the NDE technique, which depends on the thermal time history at a point. 


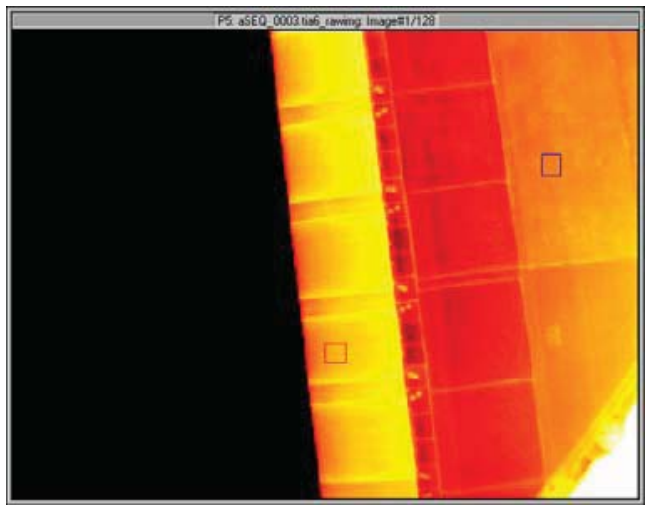

(A)

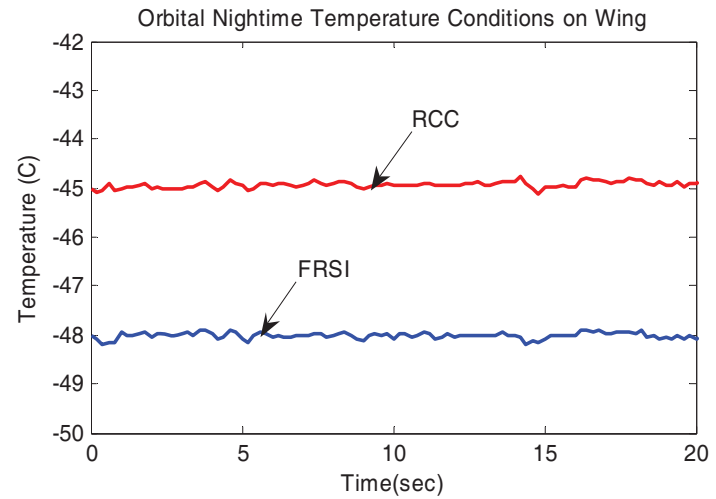

(B)

Figure 16. (A) Infrared image from Shuttle wing leading edge taken at 07:16 CDT on July 15, 2006. Orbital sunrise will occur in approximately 5 minutes. (B) Temperature versus time plots for two regions of the infrared image. The first region of interest is over the RCC panel, the second is over the white thermal blanket on the top of the wing acreage.

Using a one dimensional model and assuming the time derivative of the temperature is zero, it is possible to estimate the back side of the panel at the point of measurement is radiating into an average background temperature of $-40^{\circ} \mathrm{C}$. As would be expected the temperature is position-dependent, radiating on average into a higher temperature at the base of the panel, where it is closer to the rest of the wing and lower at the apex of the RCC panels, where the heat transfer is dominated by the inner surface of the RCC panel. Since the NDE technique is dominated by the time history at a given point, again these small variations do not significantly impact the NDE technique.

\subsection{Flight Test Objective \#2:}

The second flight test objective was to illustrate the effectiveness of the NDE technique during orbital conditions, validating the ground predictions of the on-orbit system. Four samples of RCC with known defects were flown. Two of these samples were flown in the cargo bay of STS-121 (July 12, 2006), and two were flown in a Test Article Assembly during an International Space Station (ISS) stage EVA (September 15, 2006) conducted between Shuttle flights STS-121 and STS-115. For each of these two flight tests, the two pre-damaged RCC samples included a sample with impact-induced damage and an "NDE Standard" sample. A simple NDE standard with well characterized material variation is fabricated by removing material from the back surface of the sample to specified depths and diameters.

For the samples flown on STS-121 (shown in Figure 17), the NDE standard included flatbottom holes removing $50 \%$ thickness of the material in circular areas with diameters ranging from 1 inch to 2 inches. This approximates delaminations in the RCC near the center of the through-thickness dimension. Diameters of the flat-bottom holes were chosen to approximate delamination sizes of interest to the RCC damage assessment team at the 
time the samples were fabricated. The sample with impact damage flown on STS-121 had been extensively inspected by both thermography and x-ray CT prior to the flight. This sample was determined to have an approximately $6.35 \mathrm{~cm}$ x $8.25 \mathrm{~cm}$ oblong delamination and included a small (1.25 cm long) region of Inner Mold Line (IML) coating loss. No front surface, or Outer Mold Line (OML), damage was present in either sample.

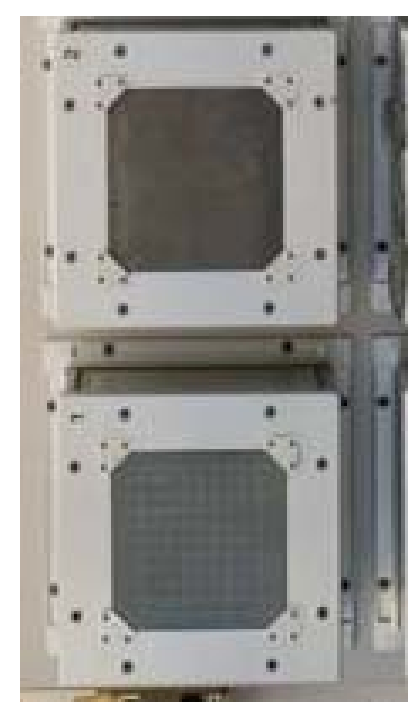

(A)

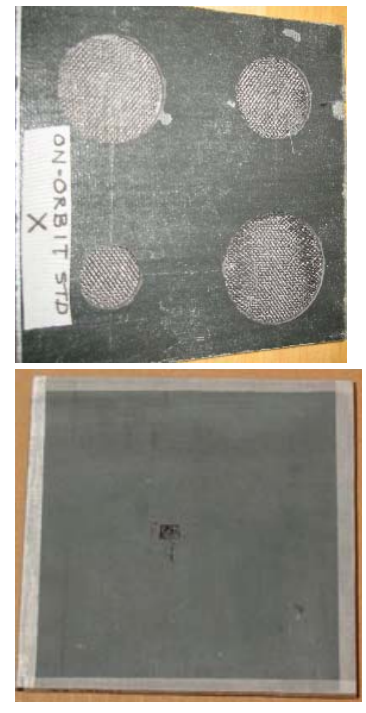

(B)

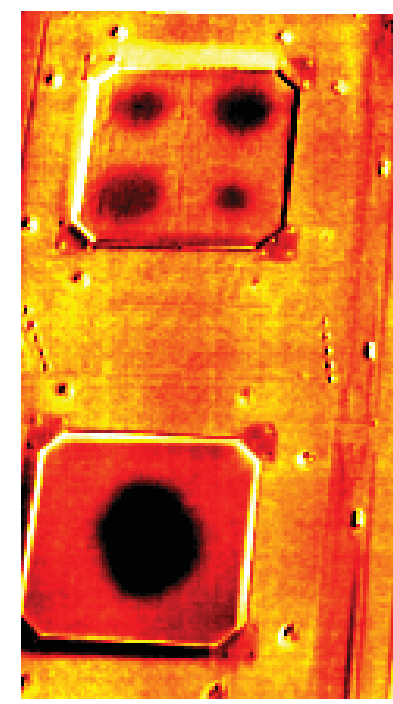

(C)

Figure 17. (A) Photograph of the OML surface of the two DTO-851 RCC samples in the Shuttle cargo bay. No OML surface damage is visible on either sample. (B) Photographs of the IML surface of the same two samples. The upper sample contains four flat-bottom-hole regions where $50 \%$ of the sample material has been removed. Diameters of the four flat-bottom holes are $2.54 \mathrm{~cm}, 3.81 \mathrm{~cm}, 4.45 \mathrm{~cm}$ and $5.08 \mathrm{~cm}$ respectively. The lower sample in the photograph shows the back surface damage due to a foam impact.

A1.27 cm square piece of the inner coating has been blown out due to the impact, and a vertical crack can be seen emanating from the damage. Ground NDE inspections revealed an oblong delamination region measuring $6.35 \mathrm{~cm} \times 8.25 \mathrm{~cm}$. (C) Processed infrared NDE imagery from STS-121 flight test. All delaminations and flat bottom holes are clearly visible.

A second flight test of the camera was conducted during a stage EVA on the ISS (Figure 18) and resulted in detection of defects approximately $2.54 \mathrm{~cm}$ in diameter created from panel impact and 4 flat-bottom-hole simulated damage regions ranging in size from 2.54 $\mathrm{cm}$ in diameter to $5.08 \mathrm{~cm}$ in diameter with $25 \%$ material loss from the back surface. The frame rate for this test was lowered to the minimum camera setting of $0.6 \mathrm{~Hz}$ to collect the maximum elapsed time of temperature data of 16.67 minutes. The sample was shaded partway through the data sequence by an ISS assembly. Spatial temperature gradients of over $17^{\circ} \mathrm{C}$ were observed. The final flight test of the camera collected additional data of the wing leading edge of the Shuttle during the daytime cycle of the orbit, providing additional data for determination of thermal boundary conditions during flight. 


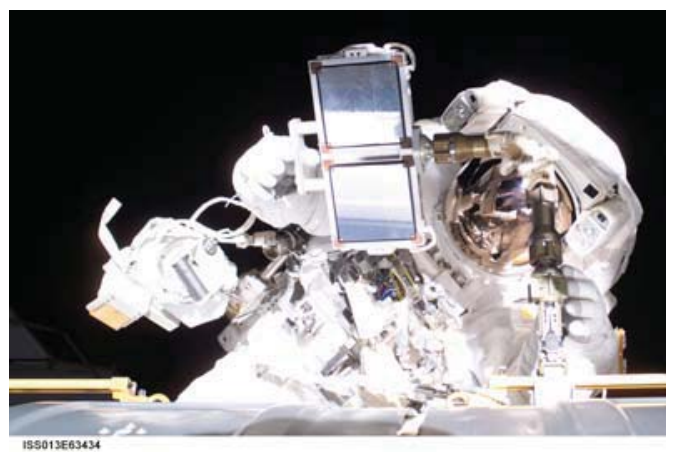

(A)

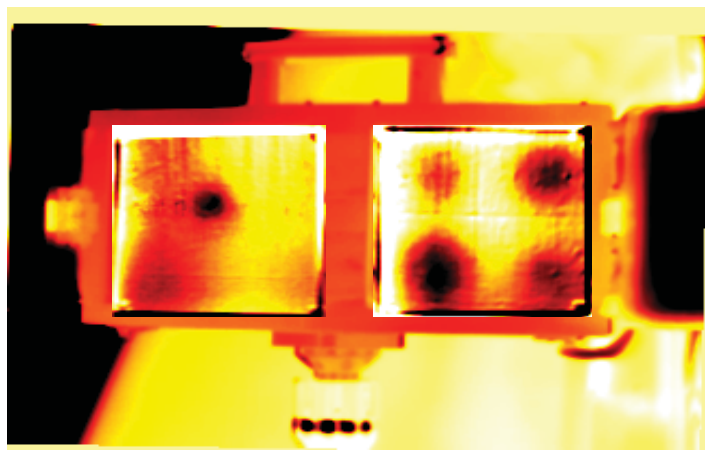

(B)

Figure 18. (A) Photograph during September 15, 2006 ISS stage EVA. Astronaut Thomas Reiter prepares the Test Article Assembly for data acquisition. The EVA IR Camera can be seen attached to his tool belt on his right side. (B) Defects detected include an approximately 1 inch diameter delamination created from panel impact and 4 flat-bottom-hole simulated damage regions ranging in size from $2.54 \mathrm{~cm}$ in diameter to $5.08 \mathrm{~cm}$ in diameter with $25 \%$ material loss from the back surface.

\section{Conclusions}

The EVA IR Camera System completed three flight tests and operated successfully each time it was used in orbit. The camera has acquired over 450 Mbytes of on-orbit infrared imagery. All mission objectives have been met, and both the camera and the inspection procedure have been effectively demonstrated. A mature NDE technology for detecting subsurface damage in RCC on the ground has been successfully transitioned and demonstrated as an orbital inspection technique. It has been shown that subsurface delaminations as small as 1 inch in diameter and $75 \%$ deep in the RCC panel can be detected with this camera system using passive solar heating. This does not represent the limit of detectability for the system, simply what has been detected to-date.

To minimize the power, weight and cost requirements for the on-orbit system, the EVA IR Camera project team conducted a series of tests on the ground that demonstrated that sunlight and shadowing could be used to create an adequate surface normal thermal gradient in RCC panels. This significantly simplified the development task and reduced project costs. However, it also increases operational complexity, because crewmembers are constrained to inspecting the RCC in sunlight.

A space flight capable IR camera is a potential tool for inspections of future exploration mission vehicle structures. The IR EVA Camera was heavily leveraged from existing technology and expertise based on extensive thermal NDE experience with ground inspections of RCC. The Shuttle Program Office established a budget of $\$ 6.4 \mathrm{M}$ for development of the EVA IR Camera system and completion of the three flight tests. The EVA IR Camera system development was completed in 9 months - in time to carry a system on STS-114 (early) and on budget. 


\section{References}

Cramer, K.E. and Winfree,W.P., "The Application Of Infrared Thermographic Inspection Techniques To The Space Shuttle Thermal Protection System," Ensayos No Destructivos Y Estructurales, pp. 227-233 (2005).

Cramer,K.E., Winfree, W.P., Hodges, K., Koshti, A, Ryan, D. and Reinhardt,W.W, "Status of Thermal NDT of Space Shuttle Materials at NASA,” Proc. SPIE Vol. 6205, pp. 1B1-9, Thermosense XXVIII (2006).

Gazarik, Michael J., Johnson, Kist, Novak, Antill, Haakenson, Howell, Pandolf, Jenkins, Yates, Stephan, Hawk, Amoroso, "Development of an extra-vehicular (EVA) infrared (IR) camera inspection system," Proc. SPIE Vol. 6205, Thermosense XXVIII; Jonathan J. Miles, G. Raymond Peacock, Kathryn M. Knettel; Eds., 2006.

Howell, Patricia A. and Zalameda, Joseph N., "Computational Analysis for Thermal NDE of Composites," Proc. SPIE Vol. 5191, pp. 18-26, Optical Diagnostics for Fluids, Solids, and Combustion II; Patrick V. Farrell, Fu-Pen Chiang, Carolyn R. Mercer, Gongxin Shen; Eds., 2003.

Jahne, Bernd, Practical Handbook on Image Processing for Scientific Applications, CRC Press, New York, 1997.

Rajic, N., "Principal component thermography for flaw contrast enhancement and flaw depth characterization in composite structures", Composite Structures, Vol. 58, pp. 521$528,2002$.

Rajic, N. "Principal Component Thermography,” DSTO-TR-1298, 2002.

Winfree, W.P., and Zalameda, J.N., "Thermographic determination of delamination depth in composites", Proc. SPIE Vol. 5073, pp. 363-373, Thermosense XXV, K. Elliott Cramer, Xavier P. Maldague; Eds., 2003.

Zalameda, J.N., Howell, P.A. and Winfree, W.P., "Compression techniques for improved algorithm computational performance”, Proc. SPIE Vol. 5782, pp. 399-406, Thermosense XXVII, G. Raymond Peacock, Douglas D. Burleigh, Jonathan J. Miles; Eds., 2005. 